\title{
Measurement of serum carboxyterminal cross-linked telopeptide of type I collagen concentration in dogs with osteosarcoma
}

\author{
John G. Hintermeister, DVM; Pamela D. Jones, DVM; Walter E. Hoffmann, DVM, PhD; \\ Arthur M. Siegel, DVM, PhD; Nikolaos G. Dervisis, DVM; Barbara E. Kitchell, DVM, PhD
}

\begin{abstract}
Objective - To evaluate the usefulness of carboxyterminal cross-linked telopeptide of type I collagen (ICTP) concentrations for screening dogs for the presence of osteosarcoma.

Sample Population-32 client-owned dogs with osteosarcoma (27 dogs with osteosarcoma of the appendicular skeleton and 5 dogs with osteosarcoma of the axial skeleton) and 44 non-tumor-bearing control dogs.

Procedures-Serum was obtained from blood samples collected from dogs with osteosarcoma and from clinically normal dogs. The serum ICTP concentration was determined by use of a commercially available radioimmunoassay for ICTP.

Results-Mean \pm SD serum ICTP concentration in the tumor-bearing dogs was 7.32 $\pm 2.88 \mathrm{ng} / \mathrm{mL}$, and in clinically normal dogs, it was $6.77 \pm 2.31 \mathrm{ng} / \mathrm{mL}$; values did not differ significantly. Mean serum ICTP concentration in dogs with appendicular osteosarcoma, compared with that of clinically normal dogs, was not significantly different. Mean serum ICTP concentration in dogs with axial skeletal tumor location was 10.82 $\pm 2.31 \mathrm{ng} / \mathrm{mL}$, compared with a value of $6.73 \pm 2.28 \mathrm{ng} / \mathrm{mL}$ in dogs with appendicular osteosarcoma.

Conclusions and Clinical Relevance-On the basis of the results of this study, serum ICTP concentrations are not a clinically useful screening tool for the detection of appendicular osteosarcoma in dogs. Despite the observation that serum ICTP concentration was higher in dogs with axial osteosarcoma than in clinically normal dogs, serum ICTP concentration determination is not a suitable screening test for osteosarcoma. (Am J Vet Res 2008;69:1481-1486)
\end{abstract}

$\mathrm{B}$ one is a dynamic tissue that undergoes constant remodeling in response to physical load and metabolic demands. The accurate measurement of bone resorption is of interest in many disease processes, including osteoporosis, osteitis deformans (Paget disease of bone), various endocrine disorders, and metastatic bone cancer. ${ }^{1-4}$ The degree of bone turnover can be evaluated both at the time of diagnosis and during the course of treatment to monitor patient response.

Type I collagen is the most abundant form of collagen in the body. It forms about $90 \%$ of the organic mass of bones and tendons and is the major collagen of many structures in the body, such as skin, cornea, and ligaments. ${ }^{5}$ Many types of cells produce type I collagen, but most is produced by fibroblasts, odontoblasts, and

\footnotetext{
Received November 14, 2007.

Accepted February 28, 2008.

From the Department of Veterinary Clinical Medicine, College of Veterinary Medicine, University of Illinois, Urbana, IL 61802. Dr. Hintermeister's present address is Texas Veterinary Oncology, 7958 Shoal Creek Blvd, Austin, TX 78759. Dr. Jones's present address is Southwest Veterinary Oncology, 86 W Juniper Ave, Ste 5, Gilbert, AZ 85233. Drs. Dervisis and Kitchell's present address is D208 Veterinary Medical Center, College of Veterinary Medicine, Michigan State University, East Lansing, MI 48824.

Address correspondence to Dr. Kitchell.
}

\begin{tabular}{ll}
\hline \multicolumn{1}{c}{ AbBreviations } \\
$\mathrm{Cl}$ & $\begin{array}{c}\text { Confidence interval } \\
\text { Carboxyterminal cross-linked telopeptide of } \\
\text { ICTP }\end{array}$ \\
& type I collagen
\end{tabular}

osteoblasts. Type I collagen is important in tissue healing and remodeling; one of the major roles of type I collagen is contribution to certain bone glycoproteins and hydroxyapatite crystals, where it is critical to handling physical stresses.

Carboxyterminal cross-linked telopeptide of type I collagen is a degradation product of bone resorption. The formation of this degradation product is mediated by specific proteinases that are released by osteoclasts. ${ }^{6}$ This activity leads to free and peptide-bound metabolites of the type I collagen. These products are released from either the N-terminal region or aminoterminal region of type I collagen. Creation of ICTP and the amino-terminal region from the parent collagen can occur as a result of the separate collagenolytic pathways that are involved in the release of these fragments, which results in the formation of different epitopes. The amino-terminal region is released during bone resorption and is dependent on cathepsin $\mathrm{K}$ activity, whereas the ICTP epitope is released following metalloproteinase activity; thus, ICTP is also 
classified as the metalloproteinase-amino-terminal region. ${ }^{7,8}$

These metabolites can be measured in the urine or serum by use of a variety of assays. The measurement of ICTP is performed only in serum, whereas assessment of the amino-terminal region uses both serum and urine. ${ }^{9}$ There are several potential contributors to the serum ICTP concentration, including diurnal variation, age, sex, and possibly diet. ${ }^{10-13}$ The cleaved ICTP molecule does not undergo further breakdown and therefore is a stable indicator of bone resorption. The measurement of ICTP has become a useful and important monitoring tool in various bone conditions in humans, such as in rheumatoid arthritis, during pregnancy, and in metastatic bone carcinoma. ${ }^{3,14-18}$ However, this assay has not yet been used to evaluate bone resorption in canine cancer patients.

Osteosarcoma is the most common primary bone tumor in dogs, usually occurring in large- to giant breed-dogs and most commonly affecting the metaphyseal region of appendicular bones. The exact etiology of osteosarcoma in dogs is unknown, and many factors for tumor development have been postulated. Rapid early growth and stress on limbs, with injury to sensitive cells, are often cited as possible reasons for bone tumor formation. The radiographic appearance of these tumors typically indicates concurrent cortical bone lysis and proliferation within the lesion. ${ }^{19}$ The standard treatment for osteosarcoma of the appendicular skeleton (ie, appendicular osteosarcoma) in dogs is amputation of the affected limb combined with adjuvant chemotherapy. Protocols that combine these elements have been shown to extend the median survival time to about 1 year in affected dogs. ${ }^{20-23}$ Most of the dogs die of distant metastatic disease. Osteosarcomas that form in the axial skeletal regions (ie, axial osteosarcoma) are commonly treated with surgical removal with or without adjuvant radiation therapy or chemotherapy for incompletely removed lesions. ${ }^{24-28}$ There are several reports ${ }^{8,9,29-37}$ in the veterinary literature regarding evaluation of various markers of bone activity and reports of markers of bone metabolism for specific diseases.

The goal of the study reported here was to evaluate the concentrations of ICTP in tumor-bearing dogs and to compare these concentrations with those detected in clinically normal dogs. In this study, we determined the serum ICTP concentration in dogs with osteosarcoma before therapeutic intervention to assess the usefulness of this test as a diagnostic screening tool. Our hypothesis was that serum ICTP concentrations are higher in tumor-bearing dogs as a result of increased bone resorption, compared with those of clinically normal dogs.

\section{Materials and Methods}

Animals-Thirty-two client-owned dogs were admitted to the Cancer Care Clinic at the University of Illinois, College of Veterinary Medicine for evaluation of suspected osteosarcoma. Lesions were identified by findings on physical examination in dogs with appendicular lesions and on radiographic and computerized tomographic images of the affected sites in dogs with axial skeletal lesions. All of the dogs $(n=32)$ had histologic confirmation of the bone lesion as an osteosar- coma or cytologic confirmation of bone sarcoma. All samples for evaluation of ICTP were obtained prior to any therapeutic intervention.

Dogs from which control samples $(n=44)$ were obtained were client-owned dogs that were admitted to the University of Illinois Veterinary Teaching Hospital for diagnosis and treatment of other disorders or were student-owned dogs that were clinically normal. Control dogs that were admitted for other conditions were determined to be free of orthopedic problems on physical examination. Selection of the control patients that were age, breed, and sex matched was attempted. All of the control patients were reevaluated at 8 months following the completion of this study and were found to be tumor free. The protocol for sample acquisition was reviewed and approved by the Laboratory Animal Care and Use Committee, and all samples were obtained with informed client consent.

Sample collection-Blood samples were collected via jugular venipuncture from all dogs. The blood was collected in a 20-mL syringe and then transferred to a glass tube for clot formation. Tubes were centrifuged at $1,252 \times \mathrm{g}$ for 10 minutes. Serum was harvested and placed in polypropylene cryovials. Samples were frozen and stored at $-70^{\circ} \mathrm{C}$. All samples were processed and placed in the freezer within 60 minutes of collection. Blood sample collection as described here is similar to a collection method reported. ${ }^{8}$

Analytical methods-Serum ICTP concentration was determined by use of a commercial radioimmunoassay. ${ }^{a}$ Briefly, in this assay, the ICTP in the unknown sample competed with iodine 125-labeled ICTP for binding sites on a rabbit polyclonal antibody against ICTP. A gamma counter detected the amount of bound tracer in the sample. The amount of unbound (free) tracer was inversely proportional to the concentration of ICTP in the test sample. The concentration of ICTP in the test sample was determined against a standard curve. This assay is used to detect serum ICTP concentrations in humans in the range of 0 to $50 \mathrm{ng} / \mathrm{mL}$, with a reported sensitivity of $0.5 \mathrm{ng} / \mathrm{mL}$. Validity of the ICTP immunoassay for use with canine serum has been described by others. ${ }^{8}$ Samples from all dogs were analyzed in duplicate by use of the same assay kit.

Statistical analysis-Continuous variables (age and serum ICTP concentration) were tested by use of the D'Agostino-Pearson test to determine whether they were normally distributed. Serum ICTP concentrations were compared among dogs with osteosarcoma and control dogs with a $t$ test for independent samples. The Pearson $\chi^{2}$ test was used to determine whether the distribution of categoric variables differed among groups. A 1-way ANOVA was used to compare the serum ICTP concentration among dogs with appendicular osteosarcoma, dogs with axial osteosarcoma, and control dogs. The Levene test for equality of variances was used to evaluate the variances among groups. A Student-Newman-Keuls test for pairwise comparisons was used to test for differences in mean serum ICTP concentrations among groups (appendicular osteosarcoma, axial osteosarcoma, and control dogs). The standard statistical definition of outliers was applied. Outliers were defined 
Table 1-Demographic characteristics of dogs in the study.

\begin{tabular}{|c|c|c|c|c|c|c|c|c|c|c|c|c|}
\hline \multirow[b]{3}{*}{ Dog group } & \multicolumn{12}{|c|}{ No. of dogs (\%) } \\
\hline & \multicolumn{2}{|c|}{ Sex } & \multicolumn{4}{|c|}{ Age in years } & \multicolumn{6}{|c|}{ Breed } \\
\hline & Male & Female & $<2$ & 2 to 5 & $>5$ to 10 & $>10$ & Rottweiler & Greyhound & $\begin{array}{l}\text { Labrador } \\
\text { Retriever }\end{array}$ & $\begin{array}{c}\text { Golden } \\
\text { Retriever }\end{array}$ & Mixed & Other \\
\hline $\begin{array}{l}\text { OSA }(n=32) \\
\text { Control }(n=44)\end{array}$ & $\begin{array}{l}19(59.4) \\
15(34.1)\end{array}$ & $\begin{array}{l}13(40.6) \\
29(65.9)\end{array}$ & $\begin{array}{l}0(0) \\
1(2.3)\end{array}$ & $\begin{array}{l}6(18.8) \\
9(20.5)\end{array}$ & $\begin{array}{l}21(65.6) \\
26(59.1)\end{array}$ & $\begin{array}{l}5(15.6) \\
8(18.2)\end{array}$ & $\begin{array}{l}6(18.8) \\
4(9.1)\end{array}$ & $\begin{array}{l}3(9.4) \\
2(4.5)\end{array}$ & $\begin{array}{l}5(15.6) \\
8(18.2)\end{array}$ & $\begin{array}{l}1(3.1) \\
4(9.1)\end{array}$ & $\begin{array}{l}7(21.9) \\
9(20.5)\end{array}$ & $\begin{array}{l}10(31.2) \\
17(38.6)\end{array}$ \\
\hline
\end{tabular}

OSA = Osteosarcoma.

as being mild when values were smaller than the lower quartile minus 1.5 times the interquartile range, or larger than the upper quartile plus 1.5 times the interquartile range (inner fences), and as extreme when values were smaller than the lower quartile minus 3 times the interquartile range, or larger than the upper quartile plus 3 times the interquartile range (outer fences).

Logistic regression analysis was used to examine the relationship between serum ICTP concentrations in dogs with osteosarcoma versus dogs without osteosarcoma, dogs with appendicular tumor sites versus dogs with axial tumor sites, and dogs with appendicular sites versus dogs without osteosarcoma. Variables (age, neuter status, breed, and serum ICTP concentration) potentially influencing the outcome (presence of osteosarcoma or not) were entered in a stepwise method in the regression model if they had values of $P<0.05$ and removed if they had values of $P>0.1$. The Spearman rank correlation coefficient $(\rho)$ was calculated to evaluate potential correlation between serum ICTP concentrations and age of patients and control dogs. A scatter diagram and a regression line were used to graphically describe the observed relationship. A residual plot was used to aid the visual evaluation of the goodness of fit of various models, and the best fitted model was chosen.

All reported $P$ values are 2 -sided. Values of $P<0.05$ were considered significant. Statistical analyses were performed with standard statistical software. ${ }^{\mathrm{b}}$

\section{Results}

Tumor-bearing dogs had a median age of 8 years (range, 2 to 12 years) with a mean of 8.16 years. Control dogs had a median age of 7 years (range, 1 to 15 years) with a mean of 7.8 years (Table 1 ). Mean \pm SD serum ICTP concentrations in the tumor-bearing dogs was $7.32 \pm 2.88 \mathrm{ng} / \mathrm{mL}$ with a $95 \% \mathrm{CI}$ of 6.33 to 8.41 $\mathrm{ng} / \mathrm{mL}$. Mean serum ICTP concentration of control dogs was $6.77 \pm 2.31 \mathrm{ng} / \mathrm{mL}$ with a $95 \% \mathrm{CI}$ of 6.07 to $7.47 \mathrm{ng} / \mathrm{mL}$ (Figure 1). The difference between the mean serum ICTP concentration in tumor-bearing dogs versus control dogs was not significant $(P=0.31)$.

Mean serum ICTP concentrations in dogs with appendicular osteosarcoma $(\mathrm{n}=27)$ was $6.73 \pm 2.28 \mathrm{ng} /$ $\mathrm{mL}$ with a $95 \% \mathrm{CI}$ of 5.45 to $6.49 \mathrm{ng} / \mathrm{mL}$. Mean serum ICTP concentration in dogs with axial osteosarcoma (n = 5) was $10.82 \pm 2.61 \mathrm{ng} / \mathrm{mL}$ with a $95 \%$ CI of 6.34 to $15.31 \mathrm{ng} / \mathrm{mL}$. The mean ICTP concentration in dogs with axial osteosarcoma was significantly $(P=0.002)$ higher than the mean serum ICTP concentration in dogs with appendicular osteosarcoma and control dogs (Figure 2).

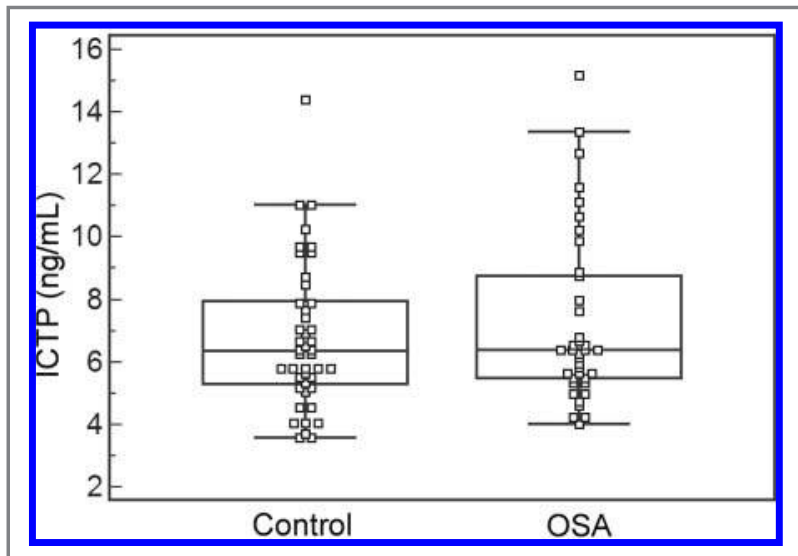

Figure 1-Box and whisker plot of serum ICTP concentrations in control and tumor-bearing dogs. The central box represents values from the lower to upper quartile (25th to 75th percentile). The middle line represents the median. A line extends from the minimum to the maximum value, excluding outliers, which are displayed as separate points. The open squares represent mild outliers, which are defined as values that are smaller than the lower quartile minus 1.5 times the interquartile range, or larger than the upper quartile plus 1.5 times the interquartile range (inner fences). OSA = Osteosarcoma.

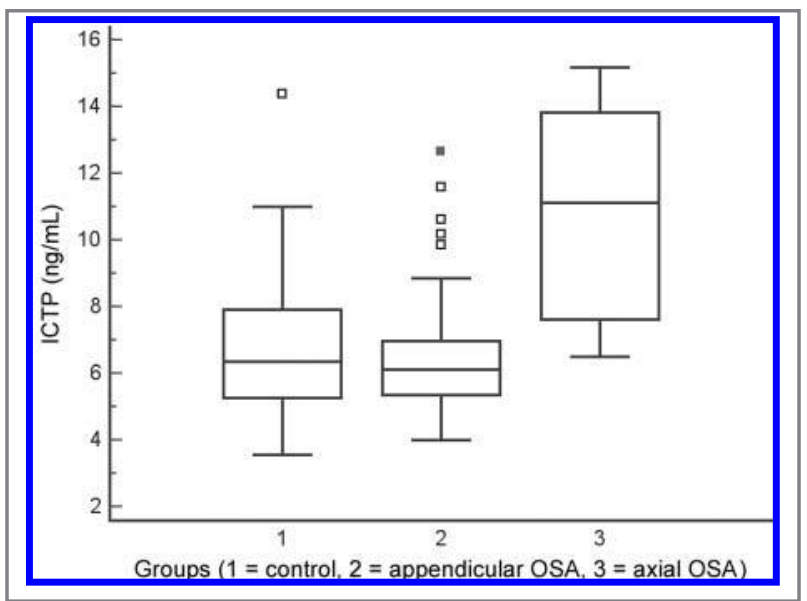

Figure 2-Box and whisker plot of serum ICTP concentrations in control dogs, dogs with appendicular osteosarcoma, and dogs with axial osteosarcoma. The filled square represents an extreme outlier value, which is defined as values that are smaller than the lower quartile minus 3 times the interquartile range, or larger than the upper quartile plus 3 times the interquartile range (outer fences). See Figure 1 for remainder of key.

Results of logistic regression analysis did not identify serum ICTP concentration as a significant determinant of outcome. Male sex was associated with in- 


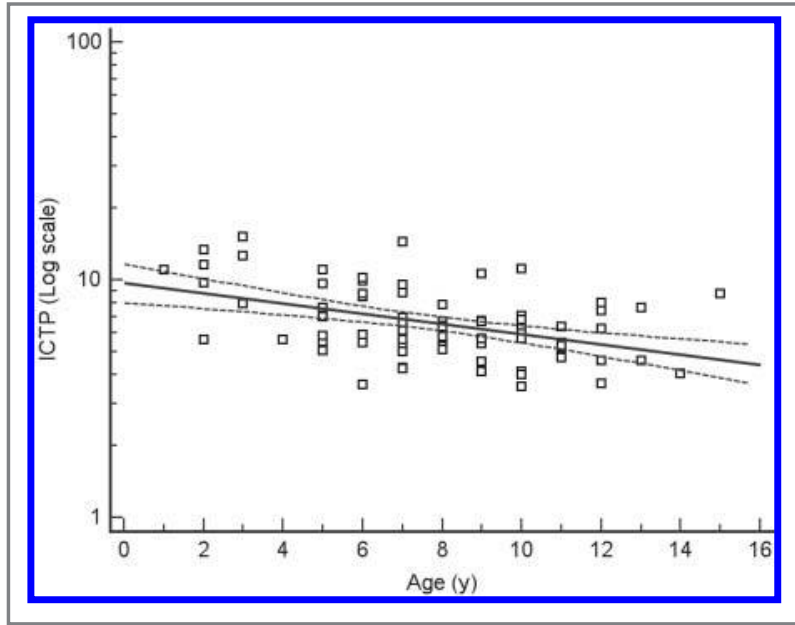

Figure 3-Regression line (solid line) and 95\% Cl (dashed lines) for the association between age and serum ICTP concentration in dogs. Serum ICTP concentration is expressed in nanograms per milliliter. Formula: Log $[$ ICTP] $=0.9,886-0.02203 x$ lage in years).

creased risk for osteosarcoma in our sample (odds ratio, 2.75; 95\% CI, 1.07 to 7.07 ).

Evaluation of the relationship between serum ICTP concentration and the age of the dog indicated a negative correlation of $-0.39(P=0.006)$. These results indicated that as the age of the individual increased, the serum ICTP concentration decreased (Figure 3). No significant difference was detected with regard to neuter status, breed, and age between dogs with osteosarcoma and control dogs.

\section{Discussion}

To our knowledge, this is the first study to evaluate serum ICTP as a bone resorption marker in dogs with osteosarcoma. There have been several studies , $^{8,29-}$ $31,36,37$ that examined the concentration of ICTP in clinically normal dogs or in dogs with pathologic conditions such as periodontal disease and osteomyelitis. The results of our study indicated that the mean serum ICTP concentration in dogs with osteosarcoma was not significantly increased, compared with that of clinically normal dogs. The serum ICTP concentration of dogs with appendicular osteosarcoma was also compared with that of clinically normal dogs, and no significant differences were found (Figure 2). Because the appendicular form of osteosarcoma makes up approximately $80 \%$ of all cases of osteosarcoma in dogs $(85 \%$ in this study), it is clear that serum ICTP concentration has no usefulness as a screening test for osteosarcoma in dogs. However, results of this study indicated that dogs with axial osteosarcoma had a higher mean serum ICP concentration than both dogs with appendicular osteosarcoma and clinically normal dogs. This suggests the possibility that serum ICTP concentration could serve as a screening test for the detection of the axial form of osteosarcoma in dogs. To confirm such a hypothesis would require a considerably larger number of dogs with osteosarcoma than was available in this study.

The precise reason for the significantly greater increase in serum ICTP concentrations in dogs with the axial form of osteosarcoma, compared with those of dogs with the appendicular form of osteosarcoma, is unknown. Although this study only has a modest sample size, the small $P$ value $(P<0.001)$ supports the possibility of a true biological difference in serum ICP concentrations between dogs with axial osteosarcoma and clinically normal dogs. There are several possible explanations for the higher serum concentrations of ICTP in dogs with axial osteosarcoma. Although it is known that $80 \%$ of the skeleton is cortical bone, there is an increased bone turnover in cancellous bone, compared with turnover in cortical bone..$^{38}$ In axial locations, the ratio of cancellous bone to cortical bone is higher, particularly in the vertebral bodies. ${ }^{39}$ It is possible that this combination of increased turnover in cancellous bone and an increased ratio of cancellous to cortical bone in axial sites may contribute to the increased concentrations of ICTP in locations of axial osteosarcomas.

In the literature, a diurnal variation in markers of bone metabolism has been identified in dogs. ${ }^{9,31}$ Results of 1 study indicate that the mean concentrations of ICTP decrease between 8 AM and 4 PM. An initial attempt was made to standardize sample collection to a particular time of day, but because of our inability to dictate the time of the entry of a patient to the hospital and client scheduling concerns, a standard sample collection time was difficult to achieve. Also, we made no attempt to collect samples serially at different time points during the day. Blood samples collected for this study from both tumor-bearing and clinically normal dogs were obtained between the hours of 8 Am and 4 PM; it is possible that the serum ICTP concentration in control dogs was reduced as the results of a normal diurnal variation. It is unknown whether serum concentrations of ICTP are also affected by chronobiologic events in patients with osteosarcoma. It is possible that influence of timing of sample collection from patients with bone tumors would be insignificant because of loss of normal homeostasis and autonomous pathologic bone activity. Evaluation of this consideration would require a standardized sample collection time and serial sample collection from tumor-bearing and clinically normal dogs.

Other possible influences on the serum ICTP concentration could include diet. All patients were fed commercial diets, and there was no attempt to standardize the diets for the tumor-bearing dogs or for control dogs. The exact influence of different diets for these patients is not known and would require additional evaluation.

The concentration of ICTP declined with advanced age in both control and tumor-bearing dogs, which is consistent with findings in a previous study. ${ }^{8}$ Age and the serum ICTP concentration were evaluated to negate the possibility of increased bone resorption activity in a geriatric control population arising as a result of other bone disorders such as osteoarthritis. The results of this study confirm that as dogs age, serum ICTP concentrations decline. Control dogs were not radiographically screened for osteoarthritis or occult neoplasia. However, follow-up of control dogs revealed dogs with osteosarcoma at 8 months after blood collection for the study.

There was an attempt to select control dogs to match our accrued tumor-bearing dogs. Tumor-bearing dogs 
were accrued sequentially as admitted to the teaching hospital for evaluation and treatment. The clinical nature of tumor-bearing dogs was random. However, control dogs that were available to us were predominately volunteered as student-owned dogs, which limited our formal matching strategy. Thus, our attempt to match dogs on the basis of age, breed, and sex between the 2 groups was largely hampered by the clinical nature of the study. The lack of appropriately matched control dogs is a limitation of this study.

The findings of this study differ from that of a study $^{34}$ on the evaluation of osteosarcoma in dogs in which the amino-terminal fragment of type I collagen or N-terminal telopeptide in urine was measured. In that study, baseline urine N-terminal telopeptide concentrations in dogs with appendicular osteosarcoma were increased, compared with those of clinically normal dogs. An additional finding in that study was a decrease in urine N-terminal telopeptide concentration with treatment of osteosarcoma. Our current study did not attempt to correlate serum ICTP concentrations with response to treatment. Evaluation of changes in serum ICTP concentrations with treatment of osteosarcoma would merit additional evaluation.

Simultaneous evaluation of multiple bone markers for bone resorption, such as ICTP, N-terminal telopeptide, and C-terminal telopeptide, in a patient population with malignant bone disease should be conducted to validate the usefulness of each individual marker in the context of this tumor. In addition, the use of markers specific for bone formation, such as osteocalcin and bone-specific alkaline phosphatase concentrations, could also be assessed to better define the usefulness of such markers in dogs with osteosarcoma.

The use of a serum concentration of a bone marker as a screening test for osteosarcoma in dogs could be beneficial for several reasons. An effective screening test could result in an earlier diagnosis of osteosarcoma in dogs at risk, which might provide clinical benefit. A screening test could allow for an earlier detection of this painful malignancy and thus might provide a window for earlier intervention that could reduce the morbidity of an individual patient. A screening test that could allow for an earlier detection, perhaps even in the preclinical phase of disease, has the potential to contribute to optimization of novel and improved treatment modalities. The potential usefulness of this test as a screening tool in individual dogs or in breeds of dogs at risk for development of osteosarcoma may merit further evaluation. Longitudinal assessment of bone markers such as ICTP throughout a given life span of a dog may provide an early tumor marker for that individual. This screening concept would require extensive validation in high-risk and low-risk breeds, such as Greyhounds versus Whippets. The use of healthy, orthopedically sound dogs that are age, breed, and sex matched would be a reasonable negative control population. In addition, the use of this test to monitor response to osteosarcoma treatment would also require robust evaluation. The ability to detect subclinical progression of the disease of a patient by use of a marker of bone metabolism, such as ICTP, could potentially allow for additional treatment with potential for prolongation of the disease-free interval and survival time. Finally, development of a bench assay kit, such as an ELISA over the radioimmunoassay used in this study, would expand the appeal of serum ICTP concentration as a diagnostic tool.

Evaluation of serum ICTP concentrations in dogs that have other bone neoplastic diseases, such as metastatic carcinoma or multiple myeloma, might also be worthy of exploration as these lesions increase ICTP concentrations in human patients. ${ }^{40-42}$

a. ICTP RIA, DiaSorin, Stillwater, Minn.

b. MedCalc for Windows, version 9.3.8.0, MedCalc Software, Mariakerke, Belgium.

\section{References}

1. Seibel MJ. Biochemical markers of bone turnover part II: clinical applications in the management of osteoporosis. Clin Biochem Rev 2006;27:123-138.

2. Shankar S, Hosking DJ. Biochemical assessment of Paget's disease of bone. J Bone Miner Res 2006;21(suppl 2):22-27.

3. Izumi M, Nakanishi Y, Takayama K, et al. Diagnostic value of bone-turnover metabolites in the diagnosis of bone metastases in patients with lung carcinoma. Cancer 2001;91:1487-1493.

4. Koga H, Naito S, Koto S, et al. Use of bone turnover marker, pyridinoline cross-linked terminal telopeptide of type I collagen (ICTP), in the assessment and monitoring of bone metastasis and prostate cancer. Prostate 1999;39:1-7.

5. Von der Mark K. Structure, biosynthesis, and gene regulation of collagens in cartilage and bone. In: Seibel MJ, Robins SP, Bilezikian JP, eds. Dynamics of bone and cartilage metabolism. San Diego: Academic Press Inc, 1999;3-29.

6. Robins SP. Fibrillogenesis and maturation of collagens. In: Seibel MJ, Robins SP, Bilezikian JP, eds. Dynamics of bone and cartilage metabolism. San Diego: Academic Press Inc, 1999;31-42.

7. Brixen K, Eriksen EF. Validation of biochemical markers of bone turnover. In: Seibel MJ, Bilezikian JP, Robins SP, eds. Dynamics of bone and cartilage metabolism: principles and clinical applications. San Diego: Academic Press Inc, 2006;83-594.

8. Seibel MJ, Eastell R, Gundberg CM, et al. Biochemical markers of bone metabolism. In: Bilezikian JP, Raisz LG, Rodan GA, eds. Principles of bone biology. San Diego: Academic Press Inc, 2002;1543-1571.

9. Allen MJ. Biochemical markers of bone metabolism in animals: uses and limitations. Vet Clin Pathol 2003;32:101-113.

10. Allen MJ, Hoffmann WE, Richardson DC, et al. Serum markers of bone metabolism in dogs. Am J Vet Res 1998;59:250-254.

11. Liesegang A, Reutter R, Sassi ML, et al. Diurnal variation in concentrations of various bone markers of bone metabolism in dogs. Am J Vet Res 1999;60:949-953.

12. Liesegang A, Risteli J, Wanner M, et al. The effects of first gestation and lactation on bone metabolism in dairy goats and milk sheep. Bone 2006;38:794-802.

13. Liesegang A, Bürgi E, Sassi ML, et al. Influence of a vegetarian diet versus a diet with fishmeal on bone in growing pigs. $J$ Vet Med A Physiol Pathol Clin Med 2002;49:230-238.

14. Iwamoto J, Takeda T, Ichimura S. Urinary cross-linked N-telopeptides of type I collagen levels in patients with rheumatoid arthritis. Calcif Tissue Int 2003;72:491-497.

15. Hellmeyer L, Ziller V, Anderer G, et al. Biochemical markers of bone turnover during pregnancy: a longitudinal study. Exp Clin Endocrinol 2006;114:506-510.

16. Costa L, Demers LM, Gouveira-Oliveria A, et al. Prospective evaluation of the peptide-bound college type I cross-n-telopeptide and c-telopeptide in predicting bone metastases status. J Clin Oncol 2002;20:850-856.

17. Kobayashi T, Gabazza EC, Taguchi O, et al. Type I collagen metabolites as tumor markers in patients with lung carcinoma. Cancer 1999;85:1951-1957.

18. Ebert W, Muley TH, Herb KP, et al. Comparison of bone scintigraphy and bone markers in the diagnosis of bone metastasis in lung carcinoma patients. Anticancer Res 2004;24:3193-3202. 
19. Dernell WS, Ehrhart NP, Straw RC, et al. Tumors of the skeletal system. In: Withrow SJ, Vail DM, eds. Withrow and MacEwen's small animal clinical oncology. 4th ed. St Louis: Saunders, 2007;540-582.

20. Kent MS, Strom A, London CA, et al. Alternating carboplatin and doxorubicin as adjunctive chemotherapy to amputation of limb-sparing surgery in the treatment of appendicular osteosarcoma in dogs. J Vet Intern Med 2004;18:540-544

21. Bergman PJ, MacEwen EG, Kurzman ID, et al. Amputation and carboplatin for treatment of dogs with osteosarcoma: 48 cases (1991 to 1993). J Vet Intern Med 1996;10:76-81.

22. Mauldin GN, Matus RE, Withrow SJ, et al. Canine osteosarcoma. Treatment by amputation versus amputation and adjuvant chemotherapy using doxorubicin and cisplatin. J Vet Intern Med 1988;2:177-180.

23. Berg J, Gebhardt MC, Rand WM. Effect of timing of postoperative chemotherapy on survival of dogs with osteosarcoma. Cancer 1997;79:1343-1350.

24. Heyman SJ, Diefenderfer DL, Goldschmidt MH, et al. Canine axial skeletal osteosarcoma. A retrospective study of 116 cases (1986 to 1989). Vet Surg 1992;21:304-310.

25. Matthiesen DT, Clark GN, Orsher RJ. En bloc resection of primary rib tumors in 40 dogs. Vet Surg 1992;21:201-204.

26. Pirkey-Ehrhart N, Withrow SJ, Straw RC, et al. Primary rib tumors in 54 dogs. J Am Anim Hosp Assoc 1995;31:65-69.

27. Dernell WS, Van Vechten BJ, Straw RC, et al. Outcome following treatment for vertebral tumors in 20 dogs (1986-1995). J Am Anim Hosp Assoc 2000;36:245-251.

28. Straw RC, Powers BE, Klausner J, et al. Canine mandibular osteosarcoma: 51 cases (1980-1992). J Am Anim Hosp Assoc 1996;32:257-262.

29. Breur GJ, Allen MJ, Carlson SJ, et al. Markers of bone metabolism in dogs breeds of different size. Res Vet Sci 2004;76:53-55.

30. Allen MJ, Allen LCV, Hoffman WE, et al. Urinary markers of type I collagen degradation in the dog. Res Vet Sci 2000;69:123-127.

31. Ladlow JF, Hoffman WE, Breur GJ, et al. Biological variability in serum and urinary indices of bone formation and resorption in dogs. Calcif Tissue Int 2002;70:186-193.
32. DeLaurier A, Jackson B, Pfeiffer D, et al. A comparison of methods for measuring serum and urinary markers of bone metabolism in cats. Res Vet Sci 2004;77:29-39.

33. Theyse LFH, Mol JA, Voorhout G, et al. The efficacy of the bone markers osteocalcin and the carboxyterminal cross-linked telopeptide of type I collagen in evaluating osteogenesis in a canine crural lengthening model. Vet J 2006;171:525-531.

34. Lacoste H, Fan TM, de Lorimier LP, et al. Urine N-telopeptide excretion in dogs with appendicular osteosarcoma. J Vet Intern Med 2006;20:335-341.

35. Ehrhart N, Dernell WS, Hoffman WE, et al. Prognostic importance of alkaline phosphatase activity in serum from dogs with appendicular osteosarcoma: 75 cases (1990-1996). J Am Vet Med Assoc 1998;213:1002-1006.

36. Philipov JP, Pascalev MD, Aminkov BY, et al. Changes in serum carboxyterminal telopeptide of type I collagen in an experimental model of canine osteomyelitis. Calcif Tissue Int 1995;57:152154.

37. Giannobile WV, Lynch SE, Denmark RG, et al. Crevicular fluid osteocalcin and pyridinoline cross-linked carboxyterminal telopeptide of type I collagen (ICTP) as markers of rapid bone turnover in periodontitis. A pilot study in beagle dogs. J Clin Periodontol 1995;22:903-910.

38. Guo XE. Mechanical properties of cortical bone and cancellous bone tissue. In: Cowin SC, ed. Bone mechanics handbook. 2nd ed. Boca Raton, Fla: CRC Press, 2001;10-13.

39. Recker RR, Barger-Lux J. Embryology, anatomy and microstructure of bone. In: Coe FL, Favus MJ, eds. Disorders of bone and mineral metabolism. Philadelphia: Lippincott Williams \& Wilkins, 2002;183.

40. Berger CE, Kroner A, Steigler H, et al. Elevated levels of serum type I collagen C-telopeptide in patients with rapidly destructive osteoarthritis of the hip. Int Orthop 2005;29:1-5.

41. Terpos E. Biochemical markers of bone metabolism in multiple myeloma. Cancer Treat Rev 2006;32(suppl 1):15-19.

42. Fonseca R, Trendle MC, Leong T, et al. Prognostic value of serum markers of bone metabolism in untreated multiple myeloma patients. Br J Haematol 2000;109:24-29. 